\title{
Optical full Hadamard matrix multiplexing and noise effects: errata
}

\author{
L. Streeter, ${ }^{1, *}$ M. J. Cree, ${ }^{1}$ and G. R. Burling-Claridge ${ }^{2}$ \\ ${ }^{1}$ School of Engineering, University of Waikato, Private Bag 3105, Hamilton 3240, New Zealand \\ ${ }^{2}$ FullSpectrum Ltd., Hamilton, New Zealand \\ ${ }^{*}$ Corresponding author: Ivs2@ phys.waikato.ac.nz \\ Received 27 September 2011; \\ posted 27 September 2011 (Doc. ID 153800); published 4 November 2011
}

\begin{abstract}
The model for Poisson random noise under Hadamard multiplexing is revised. The new model accounts for the variation of the Hadamard multiplexed measurements, as well as the previously considered variation due to Poisson fluctuations. A numerical simulation matches the model prediction within uncertainty. (C) 2011 Optical Society of America

OCIS codes: $\quad 030.4280,350.6980,110.4234,110.1758$.
\end{abstract}

In [1] we analyzed the Hadamard $H$-matrix for use in multiplexing on an optical imaging spectrometer. As part of the analysis we made a prediction for the noise level at decoding due to Poisson noise in the signal at acquisition, which, we now realize, underestimated the size of the Poisson noise due to multiplexing. Here we provide a revised formula with support from a numerical simulation.

In [1] complement encoding was employed to implement a full $H$-matrix, $H$, in an optical setting. Let $\mathbf{p}$ be data to be determined from measurements a where $\mathbf{a}=H \mathbf{p}$. The $H$-matrix has both positive and negative entries, but in incoherent optics only positive values are physically realizable. Complement encoding solves the problem by taking two sets of measurements, where the first set of measurements are taken according to the positive entries in the $H$-matrix, $\mathbf{a}^{+}=H^{+} \mathbf{p}$, and the second set according to the negative entries $\mathbf{a}^{-}=H^{-} \mathbf{p}$, where $H^{ \pm}=$ $\pm \frac{1}{2}(H \pm 1)$ and $H=H^{+}-H^{-}$. The final encoded measurement is computed by

$$
\mathbf{a}=\mathbf{a}^{+}-\mathbf{a}^{-}=H \mathbf{p}
$$

0003-6935/11/326092-02\$15.00/0

(C) 2011 Optical Society of America
Now assume that the illumination is a Poisson random variable $r$ with mean and variance $\langle r\rangle$. The entries in $\mathbf{p}$ represent attenuation of the light. Thus the variance of each element $p_{i}$ in $\mathbf{p}$ is $\sigma_{i}^{2}=\langle r\rangle p_{i}$. Herein we omit the explicit optics attenuation term not related to the object being imaged, $\alpha_{s}$, used in [1] . It was stated that each $a_{j}^{+}$entry in $\mathbf{a}^{+}$, and $a_{j}^{-}$in $\mathbf{a}^{-}$, has variance due to Poisson effects, respectively $\left(\sigma^{+}\right)^{2}$ and $\left(\sigma^{-}\right)^{2}$ given by

$$
\begin{aligned}
& \left(\sigma^{+}\right)^{2}=\delta_{j} N\langle r\rangle\langle p\rangle, \\
& \left(\sigma^{-}\right)^{2}=\left(1-\delta_{j}\right) N\langle r\rangle\langle p\rangle,
\end{aligned}
$$

where $N$ is the number of entries in $\mathbf{p},\langle p\rangle$ is the mean of the $p_{i}$, and $0 \leq \delta_{j} \leq 1$. From Eq. (3) it follows that the Poisson variance in the combined measurements $a_{j}$ in $\mathbf{a}$ is

$$
\sigma_{j}^{2}=\left(\sigma_{j}^{+}\right)^{2}+\left(\sigma_{j}^{-}\right)^{2}=N\langle r\rangle\langle p\rangle .
$$

Equation (4) appeared to adequately describe the results from the physical experiment described in [1], namely that the noise level varied with wavelength in acquired spectra. The analysis leading to the result in Eq. (4), however, only accounts for the 
variation in the illumination and neglects the fact that the measurements themselves vary.

Here in computing the variance we explicitly include the interaction of two effects:

- Poisson error with variance $\langle r\rangle$ and mean 0 (we are considering only the variation in the illumination about the mean) and

- attenuation with means $H^{+} \mathbf{p} \approx H^{-} \mathbf{p} \approx N\langle p\rangle / 2$ and variances $\sigma_{H^{+} \mathbf{p}}^{2}$ and $\sigma_{H^{-}}^{2}$, the variance over the encoded $\mathbf{p}$.

Using the equation for the product of two variables [2] the variance at measurement is

$$
\begin{gathered}
\sigma_{\mathbf{a}}^{2}=\sigma_{\mathbf{a}+}^{2}+\sigma_{\mathbf{a}_{-}}^{2} \\
\approx\left(\langle r\rangle \frac{N^{2}\langle p\rangle^{2}}{4}+\langle r\rangle \sigma_{H^{+} \mathbf{p}}^{2}\right)+\left(\langle r\rangle \frac{N^{2}\langle p\rangle^{2}}{4}+\langle r\rangle \sigma_{H^{-} \mathbf{p}}^{2}\right), \\
=\langle r\rangle \frac{N^{2}\langle p\rangle^{2}}{2}+\langle r\rangle\left(\sigma_{H^{+} \mathbf{p}}^{2}+\sigma_{H^{-} \mathbf{p}}^{2}\right) .
\end{gathered}
$$

Of greatest concern is the $N^{2}$ term in the numerator of Eq. (6), indicating that Eq. (13) of [1], paraphrased as Eq. ( 14 ) above, underestimates the Poisson noise effect.

As a specific example, consider a signal $\mathbf{p}$ of $N=8$ discrete values $p_{i}=i / N$, and let $r$ be a Poisson random variable with $\langle r\rangle=1000$. In MatLab 2009a [3], we simulated multiplexing using complement encoding 100 times, and from the simulated measurements estimated the $N$ variances at each of the $a_{j}$ in a, and then the mean of the $N$ variances. The 100 simulated measurements were repeated 100 times over, yielding 100 mean of variances, and the overall mean variance and standard deviation in the variance were calculated. The predicted variance at acquisition using Eq. (6) is 11,938, whereas the underestimated prediction from Eq. (4) is 4500. The numerical simulation gave an estimate of the variance in the acquisition of $11,700 \pm 600$, matching the predicted value within uncertainty.

In [1] we drew the conclusion that, in an optical setting, $H$-matrix multiplexing has no effect on the average photon noise level when compared to no multiplexing. The improvement expected from $H$-matrix multiplexing depends on the relative level of photon noise and random additive noise. It followed from Eq. (4) that the worst that $H$-matrix multiplexing can achieve is the same overall noise level as no multiplexing. Contrary to [1], Eq. (6) indicates that beyond a certain light level multiplexing may increase the overall noise level above that of no multiplexing. Nevertheless the conclusion drawn in [1] that $H$-matrix multiplexing produces a preferential reduction of additive noise remains true. The implications of Eq. (6) will be investigated in detail in future work.

\section{References}

1. L. Streeter, G. R. Burling-Claridge, M. J. Cree, and R. Künnemeyer, "Optical full hadamard matrix multiplexing and noise effects," Appl. Opt. 48, 2078-2085 (2009).

2. L. A. Goodman, "On the exact variance of products," J. Am. Stat. Assoc. 55, 708-713 (1960).

3. Mathworks, Massachusetts, USA. 